\title{
Mappings between the lattices of saturated submodules with respect to a prime ideal
}

\author{
Morteza Noferesti (D), Hosein Fazaeli Moghimi*(D), Mohammad Hossein Hosseini (D) \\ Department of Mathematics, University of Birjand, P.O.Box 97175-615, Birjand, Iran
}

\begin{abstract}
Let $\mathfrak{S}_{p}\left({ }_{R} M\right)$ be the lattice of all saturated submodules of an $R$-module $M$ with respect to a prime ideal $p$ of a commutative ring $R$. We examine the properties of the mappings $\eta: \mathfrak{S}_{p}\left({ }_{R} R\right) \rightarrow \mathfrak{S}_{p}\left({ }_{R} M\right)$ defined by $\eta(I)=S_{p}(I M)$ and $\theta: \mathfrak{S}_{p}\left({ }_{R} M\right) \rightarrow \mathfrak{S}_{p}\left({ }_{R} R\right)$ defined by $\theta(N)=(N: M)$, in particular considering when these mappings are lattice homomorphisms. It is proved that if $M$ is a semisimple module or a projective module, then $\eta$ is a lattice homomorphism. Also, if $M$ is a faithful multiplication $R$-module, then $\eta$ is a lattice epimorphism. In particular, if $M$ is a finitely generated faithful multiplication $R$-module, then $\eta$ is a lattice isomorphism and its inverse is $\theta$. It is shown that if $M$ is a distributive module over a semisimple ring $R$, then the lattice $\mathfrak{S}_{p}\left(R_{R} M\right)$ forms a Boolean algebra and $\eta$ is a Boolean algebra homomorphism.
\end{abstract}

Mathematics Subject Classification (2020). 13C13, 06B99, 06E99, 13C99

Keywords. saturated submodules with respect to a prime ideal, $\eta$-modules, $\theta$-modules, $\mathfrak{S}$-distributive modules, semisimple rings

\section{Introduction}

We assume throughout this paper that all rings are commutative with nonzero identity and all modules are unitary. Let $R$ be a ring and $M$ be an $R$-module. For any submodule $N$ of $M$, we denote the annihilator of the $R$-module $M / N$ by $(N: M)$, i.e., $(N: M)=$ $\{r \in R \mid r M \subseteq N\}$.

It is well-known that the collection of all submodules of $M$ forms a lattice with respect to the operations $\vee$ and $\wedge$ defined by

$$
L \vee N=L+N \text { and } L \wedge N=L \cap N .
$$

Note that this lattice, denoted $\mathcal{L}\left({ }_{R} M\right)$, is bounded with the least element $(0)$ and greatest element $M$. Recently, P.F. Smith has studied several mappings between $\mathcal{L}\left({ }_{R} R\right)$ and $\mathcal{L}\left({ }_{R} M\right)$ [22-24]. For instance, in [22], he examined conditions under which the mappings $\lambda: \mathcal{L}\left({ }_{R} R\right) \rightarrow \mathcal{L}\left({ }_{R} M\right)$ defined by $\lambda(I)=I M$ and $\mu: \mathcal{L}\left({ }_{R} M\right) \rightarrow \mathcal{L}\left({ }_{R} R\right)$ defined by $\mu(N)=(N: M)$ are injective, surjective or lattice homomorphisms. An $R$-module $M$ is called a $\lambda$-module (respectively $\mu$-module), if $\lambda$ (respectively $\mu$ ) is a lattice homomorphism.

\footnotetext{
*Corresponding Author.

Email addresses: morteza_noferesti@birjand.ac.ir (M. Noferesti), hfazaeli@birjand.ac.ir (H.F. Moghimi), mhhosseini@birjand.ac.ir (M.H. Hosseini)

Received: 12.08.2019; Accepted: 03.06.2020
} 
The study of the mappings $\lambda$ and $\mu$ continued in [23], considering when these mappings are complete lattice homomorphisms.

A proper submodule $P$ of $M$ is called a prime submodule if for $r \in R$ and $x \in M, r x \in P$ implies that $r \in(P: M)$ or $x \in P$ (see, for example, $[2,6,18,19]$ ). For a proper submodule $N$ of an $R$-module $M$, the intersection of all prime submodules of $M$ containing $N$ is called the radical of $N$ and denoted by $\operatorname{rad} N$; if there are no such prime submodules, $\operatorname{rad} N$ is $M$ (see, for example, $[11,14,17])$. A submodule $N$ of $M$ is called a radical submodule if $\operatorname{rad} N=N$. The collection of all radical submodules of $M$ which is denoted by $\mathcal{R}\left({ }_{R} M\right)$ forms a lattice with respect to the following operations:

$$
L \vee N=\operatorname{rad}(L+N) \text { and } L \wedge N=L \cap N .
$$

Note that $\mathcal{R}\left({ }_{R} M\right)$ is a bounded lattice with the least element $\operatorname{rad}(0)$ and the greatest element $M$.

In [20], H.F. Moghimi and J.B. Harehdashti have studied the properties of the mappings $\rho: \mathcal{R}\left({ }_{R} R\right) \rightarrow \mathcal{R}\left({ }_{R} M\right)$ defined by $\rho(I)=\operatorname{rad}(I M)$ and $\sigma: \mathcal{L}\left({ }_{R} R\right) \rightarrow \mathcal{L}\left({ }_{R} M\right)$ defined by $\sigma(N)=(N: M)$, in particular considering when these mappings are lattice monomorphisms or epimorphisms. Later in [9], they investigated conditions under which these mappings are complete homomorphisms. Note that $\rho$ is always a lattice homomorphism, but not necessarily a complete lattice homomorphism. An $R$-module $M$ is called a $\sigma$ module if $\sigma$ is a lattice homomorphism.

Let $M$ be an $R$-module. For a prime ideal $p$ of $R$ and a submodule $N$ of $M$, the set $S_{p}(N)=\{m \in M \mid c m \in N$ for some $c \in R \backslash p\}$ is called the saturation of $N$ with respect to $p$. It is clear that $N \subseteq S_{p}(N)$. It is said that $N$ is saturated with respect to $p$, if $N=S_{p}(N)$. It is easily seen that $S_{p}(N)$ is a saturated submodule of $M$ (see $[15,16]$, for more details about saturation of submodules). The collection of all saturated submodules of an $R$-module $M$ with respect to a fixed prime ideal $p$ of $R$ is a lattice with the following operations:

$$
L \vee N=S_{p}(L+N) \quad \text { and } \quad L \wedge N=L \cap N .
$$

We shall denote this lattice by $\mathfrak{S}_{p}(R M)$, or by $\mathfrak{S}_{p}(M)$ if there is no ambiguity about $R$. Note that $\mathfrak{S}_{p}(M)$ is bounded, with the least element $S_{p}(0)$ and the greatest element $M$.

Let $R$ be a ring, $p$ a fixed prime ideal of $R$ and $M$ an $R$-module. Now consider the mappings $\eta: \mathfrak{S}_{p}(R) \rightarrow \mathfrak{S}_{p}(M)$ defined by

$$
\eta(I)=S_{p}(I M),
$$

for every saturated ideal $I$ of $R$, and $\theta: \mathfrak{S}_{p}(M) \rightarrow \mathfrak{S}_{p}(R)$ defined by

$$
\theta(N)=(N: M),
$$

for every saturated submodule $N$ of $M$. It will be convenient for us to call the module $M$ an $\eta$-module (resp. a $\theta$-module) in case the above mapping $\eta$ (resp. $\theta$ ) is a lattice homomorphism.

In this paper, we investigate conditions under which $\eta$ and $\theta$ are lattice homomorphisms, in particular considering when $\eta$ and $\theta$ are Boolean algebra homomorphisms. It is shown that modules over Prüfer domains (Corollary 2.4), projective modules (Corollary 2.6) and semisimple $R$-modules (Corollary 2.7) are three classes of $\eta$-modules. It is proved that if $M$ is a faithful multiplication $R$-module, then $\eta$ is a lattice epimorphism, and in particular $\mathfrak{S}_{p}(M)$ is isomorphic to a quotient of $\mathfrak{S}_{p}(R)$ (Theorem 2.8) for all prime ideals $p$ of $R$. It is shown that a finitely generated module $M$ is a $\theta$-module if and only if it is a multiplication module (Corollary 2.11). In particular, every cyclic $R$-module is a $\theta$-module (Corollary 2.10). Moreover, if $M$ is a finitely generated faithful multiplication $R$-module then $\eta$ and $\theta$ are lattice isomorphisms (Corollary 2.17).

An $R$-module $M$ is called distributive if $\mathcal{L}\left({ }_{R} M\right)$ is a distributive lattice (see, for example, 
[8]). A ring $R$ is called arithmetical if it is a distributive $R$-module. We say that an $R$ module $M$ is $\mathfrak{S}$-distributive with respect to a prime ideal $p$ of $R$ if $\mathfrak{S}_{p}(M)$ is a distributive lattice. It is proved that an $R$-module $M$ is distributive if and only if it is $\mathfrak{S}$-distributive with respect to any prime ideal of $R$ (Corollary 3.4). In particular, every multiplication module over an arithmetical ring $R$ is $\mathfrak{S}$-distributive with respect to any prime ideal of $R$ (Corollary 3.5). It is shown that if $M$ is a distributive module over a semisimple ring $R$, then $\mathfrak{S}_{p}(M)$ forms a Boolean algebra (Theorem 3.7) and $\eta$ is a Boolean algebra homomorphism (Theorem 3.13). In particular, if $M$ is a multiplication module over a semisimple ring $R$, then $\eta$ is a Boolean algebra epimorphism (Corollary 3.14).

\section{2. $\eta$-modules and $\theta$-modules}

We start with a lemma which collects some facts about saturation of submodules.

Lemma 2.1. Let $R$ be a ring, $p$ a prime ideal of $R$ and $M$ an $R$-module. Then

(1) $S_{p}(L \cap N)=S_{p}(L) \cap S_{p}(N)$ for all submodules $L$ and $N$ of $M$;

(2) $S_{p}\left(S_{p}(I M)+S_{p}(J M)\right)=S_{p}\left(S_{p}(I+J) M\right)=S_{p}(I M+J M)$ for all ideals $I$ and $J$ of $R$.

Proof. (1) Clear.

(2) Since $I M \subseteq(I+J) M \subseteq S_{p}(I+J) M$, we conclude that $S_{p}(I M) \subseteq S_{p}\left(S_{p}(I+J) M\right)$. Similarly, $S_{p}(J M) \subseteq S_{p}\left(S_{p}(I+J) M\right)$. Therefore, we have $S_{p}(I M)+S_{p}(J M) \subseteq S_{p}\left(S_{p}(I+\right.$ $J) M)$. Hence we have $S_{p}\left(S_{p}(I M)+S_{p}(J M)\right) \subseteq S_{p}\left(S_{p}(I+J) M\right)$. Now, let $x \in S_{p}\left(S_{p}(I+\right.$ $J) M)$. Then there exists $c \in R \backslash p$ such that $c x \in S_{p}(I+J) M$. Therefore $c x=\sum_{i=1}^{k} r_{i} x_{i}$ for some $r_{i} \in S_{p}(I+J)$ and $x_{i} \in M(1 \leq i \leq k)$. Thus there are $c_{i} \in R \backslash p(1 \leq i \leq k)$ such that $c_{i} r_{i} \in I+J$, and so $c_{1} \ldots c_{k} c x \in(I+J) M$. It follows that $x \in S_{p}((I+J) M)$. Hence we have $S_{p}\left(S_{p}(I+J) M\right) \subseteq S_{p}(I M+J M)$. It is also clear that $S_{p}(I M+J M) \subseteq$ $S_{p}\left(S_{p}(I M)+S_{p}(J M)\right)$.

Theorem 2.2. Let $R$ be a ring, $p$ a prime ideal of $R$ and $M$ an $R$-module. Then the following statements are equivalent:

(1) $M$ is an $\eta$-module over $R$;

(2) $\quad S_{p}((I \cap J) M)=S_{p}(I M) \cap S_{p}(J M)$ for all ideals $I$ and $J$ of $R$;

(3) $\quad\left(I_{p} \cap J_{p}\right) M_{p}=I_{p} M_{p} \cap J_{p} M_{p}$ for all ideals $I$ and $J$ of $R$;

(4) $M_{p}$ is a $\lambda$-module over $R_{p}$.

Proof. (1) $\Rightarrow(2)$ By definition.

$(2) \Rightarrow(1)$ Let $I, J \in \mathfrak{S}_{p}(R)$. By the assumption, $\eta(I \wedge J)=\eta(I) \wedge \eta(J)$.

By using Lemma 2.1, we have

$$
\begin{aligned}
\eta(I \vee J) & =S_{p}((I \vee J) M)=S_{p}\left(S_{p}(I+J) M\right) \\
& =S_{p}\left(S_{p}(I M)+S_{p}(J M)\right) \\
& =S_{p}(I M) \vee S_{p}(J M) \\
& =\eta(I) \vee \eta(J) .
\end{aligned}
$$

$(2) \Rightarrow(3)$ Let $z \in I_{p} M_{p} \cap J_{p} M_{p}$. Then $z=\sum_{i=1}^{k} a_{i} x_{i} / s_{i}=\sum_{i=1}^{k} b_{i} y_{i} / t_{i}$ for some $a_{i} \in I$, $b_{i} \in J, x_{i}, y_{i} \in M, s_{i}, t_{i} \in R \backslash p$. Hence we have $s_{1} \ldots s_{k} t_{1} \ldots t_{k} z \in I M \cap J M$ which follows that $z \in S_{p}(I M) \cap S_{p}(J M)$. Therefore by (2), $z \in S_{p}((I \cap J) M)$. Thus $c z \in(I \cap J) M$ for some $c \in R \backslash p$, and so $z \in\left(I_{p} \cap J_{p}\right) M_{p}$ as desired. The reverse inclusion is clear.

$(3) \Rightarrow(2)$ Let $x \in S_{p}(I M) \cap S_{p}(J M)$. Then $c x \in I M$ and $d x \in J M$ for some $c, d \in R \backslash p$. Therefore $c x=\sum_{i=1}^{k} c_{i} x_{i}$ and $d x=\sum_{j=1}^{k} d_{j} x_{j}^{\prime}$ for some $c_{i} \in I, d_{j} \in J$ and $x_{i}, x_{j}^{\prime} \in M$ $(1 \leq i, j \leq k)$. Thus $c_{1} d x=\sum_{j=1}^{k} c_{1} d_{j} x_{j}^{\prime}$ and hence $c_{1} d x \in(I \cap J) M$ such that $c_{1} d \in R \backslash p$. Thus $x \in S_{p}((I \cap J) M)$. The reverse inclusion is clear.

$(3) \Leftrightarrow(4)$ Follows from [22, Lemma 2.1 (ii)]. 
Let $R$ be a domain with the field of fractions $K$. A non-zero ideal $I$ of $R$ is called invertible provided $I^{-1} I=R$ where $I^{-1}=\{k \in K: k I \subseteq R\}$. A domain $R$ is called Prüfer if every non-zero finitely generated ideal of $R$ is invertible (see, for more details, [13]).

Corollary 2.3. Let $R$ be a domain, $p$ a prime ideal of $R$ and $M$ an $R$-module. Then the following statements are equivalent:

(1) $R_{p}$ is Prüfer;

(2) Every $R_{p}$-module is a $\lambda$-module;

(3) Every $R$-module is an $\eta$-module.

Proof. (1) $\Leftrightarrow(2)$ By [22, Theorem 2.3].

$(2) \Leftrightarrow(3)$ By Theorem 2.2.

Corollary 2.4. Let $R$ be any Prüfer domain. Then every $R$-module is an $\eta$-module.

Proof. Let $R$ be a Prüfer domain and $p$ be a prime ideal of $R$. Then by [13, Theorem 6.6], $R_{p}$ is a valuation ring. Thus by [22, Proposition 2.4], every $R_{p}$-module is a $\lambda$-module and hence by Corollary 2.3 , every $R$-module is an $\eta$-module.

Theorem 2.5. Let $R$ be any ring. Then

(1) Every direct summand of an $\eta$-module is an $\eta$-module.

(2) Every direct sum of $\lambda$-modules is an $\eta$-module.

Proof. (1) Let $K$ be a direct summand of an $\eta$-module $M$. Let $I$ and $J$ be any ideals of $R$ and $p$ be a prime ideal of $R$. Then by Lemma 2.1 (1) and Theorem 2.2, we have

$$
\begin{aligned}
S_{p}(I K) \cap S_{p}(J K) & =S_{p}(K \cap I M) \cap S_{p}(K \cap J M) \\
& =S_{p}(K) \cap S_{p}(I M) \cap S_{p}(J M) \\
& =S_{p}(K) \cap S_{p}((I \cap J) M) \\
& =S_{p}(K \cap(I \cap J) M) \\
& =S_{p}((I \cap J) K) .
\end{aligned}
$$

Thus by Theorem $2.2, K$ is an $\eta$-module.

(2) Let $M_{i}(i \in \mathfrak{I})$ be any collection of $\lambda$-modules and let $M=\oplus_{i \in \mathfrak{I}} M_{i}$. Given any ideals $I$ and $J$ of $R$, by [22, Lemma 2.1], we have

$$
\begin{aligned}
S_{p}(I M) \cap S_{p}(J M) & =S_{p}\left(\oplus_{i \in \mathfrak{I}} I M_{i}\right) \cap S_{p}\left(\oplus_{i \in \mathfrak{I}} J M_{i}\right) \\
& =S_{p}\left(\oplus_{i \in \mathfrak{I}} I M_{i} \cap \oplus_{i \in \mathfrak{I}} J M_{i}\right) \\
& =S_{p}\left(\oplus_{i \in \mathfrak{I}}\left(I M_{i} \cap J M_{i}\right)\right) \\
& =S_{p}\left(\oplus_{i \in \mathfrak{I}}(I \cap J) M_{i}\right) \\
& =S_{p}((I \cap J) M) .
\end{aligned}
$$

Thus by Theorem 2.2, $M$ is an $\eta$-module.

Corollary 2.6. For any ring $R$, every projective $R$-module is an $\eta$-module.

Proof. By [22, Lemma 2.1], every ring $R$ is a $\lambda$-module. Thus by [10, Theorem IV.2.1] and Theorem 2.5(2), every free $R$-module is an $\eta$-module, and therefore by [10, Theorem IV.3.4] and Theorem 2.5(1), every projective $R$-module is an $\eta$-module.

Corollary 2.7. For any ring $R$, every semisimple $R$-module is an $\eta$-module.

Proof. Clearly every simple module is a $\lambda$-module. Since any semisimple module is a direct sum of a family of simple submodules, the result follows from Theorem 2.5(2). 
An $R$-module $M$ is called a multiplication module if the mapping $\lambda$ is surjective, i.e., for each submodule $N$ of $M$ there exist an ideal $I$ of $R$ such that $N=I M$. In this case, we can take $I=(N: M)$ (see, for example, $[4,7])$.

Theorem 2.8. Let $M$ be a faithful multiplication $R$-module. Then $\eta$ is a lattice epimorphism.

In particular, $\mathfrak{S}_{p}(M)$ is isomorphic to a quotient of $\mathfrak{S}_{p}(R)$ for all prime ideals $p$ of $R$.

Proof. Since $M$ is a faithful multiplication $R$-module, $M$ is a $\lambda$-module by [22, Theorem 2.12]. Thus by [22, Lemma 2.1], $(I \cap J) M=I M \cap J M$ for all ideals $I$ and $J$ of $R$. It follows that, by Lemma 2.1 (1),

$$
S_{p}((I \cap J) M)=S_{p}(I M \cap J M)=S_{p}(I M) \cap S_{p}(J M)
$$

for all ideals $I$ and $J$ and prime ideals $p$ of $R$. Hence by Theorem 2.2, $\eta$ is a lattice homomorphism. Now, let $p$ be a prime ideal of $R$ and $N \in \mathfrak{S}_{p}(M)$. Since $M$ is a multiplication module, we have

$$
\eta((N: M))=S_{p}((N: M) M)=S_{p}(N)=N
$$

and therefore $\eta$ is an epimorphism. Now, we define the relation $\sim$ on $\mathfrak{S}_{p}(R)$ by

$$
I \sim J \Leftrightarrow S_{p}(I M)=S_{p}(J M) .
$$

It is evident that $\sim$ is an equivalence relation on $\mathfrak{S}_{p}(R)$. We show that $\sim$ is a congruence relation. Assume that $I_{1} \sim J_{1}$ and $I_{2} \sim J_{2}$. Thus we have $S_{p}\left(I_{1} M\right)=S_{p}\left(J_{1} M\right)$ and $S_{p}\left(I_{2} M\right)=S_{p}\left(J_{2} M\right)$. Since $M$ is a faithful multiplication module,

$$
\begin{aligned}
S_{p}\left(\left(I_{1} \cap J_{1}\right) M\right) & =S_{p}\left(I_{1} M\right) \cap S_{p}\left(J_{1} M\right) \\
& =S_{p}\left(I_{2} M\right) \cap S_{p}\left(J_{2} M\right) \\
& =S_{p}\left(\left(I_{2} \cap J_{2}\right) M\right),
\end{aligned}
$$

and therefore $I_{1} \wedge J_{1} \sim I_{2} \wedge J_{2}$. Also, by Lemma 2.1 (2),

$$
\begin{aligned}
S_{p}\left(S_{p}\left(I_{1}+J_{1}\right) M\right) & =S_{p}\left(S_{p}\left(I_{1} M\right)+S_{p}\left(J_{1} M\right)\right) \\
& =S_{p}\left(S_{p}\left(I_{2} M\right)+S_{p}\left(J_{2} M\right)\right) \\
& =S_{p}\left(S_{p}\left(I_{2}+J_{2}\right) M\right)
\end{aligned}
$$

which follows that $I_{1} \vee J_{1} \sim I_{2} \vee J_{2}$. Thus $\mathfrak{S}_{p}(R) / \sim$, the set of equivalence classes with respect to $\sim$, is a lattice with the following operations:

$$
I / \sim \tilde{\vee} J / \sim=I \vee J / \sim \text { and } I / \sim \tilde{\wedge} J / \sim=I \wedge J / \sim .
$$

Now, the mapping $\bar{\eta}: \mathfrak{S}_{p}(R) / \sim \rightarrow \mathfrak{S}_{p}(M)$ given by $\bar{\eta}(I / \sim)=\eta(I)=S_{p}(I M)$ is a lattice isomorphism.

Recall that $\theta: \mathfrak{S}_{p}(M) \rightarrow \mathfrak{S}_{p}(R)$ defined by $\theta(N)=(N: M)$ is the restriction of the mapping $\mu: \mathcal{L}\left({ }_{R} M\right) \rightarrow \mathcal{L}\left({ }_{R} R\right)$ to $\mathfrak{S}_{p}(M)$ given in [22]. Thus every $\mu$-module is a $\theta$-module.

Theorem 2.9. Let $R$ be a ring and $M$ an R-module. Consider the following statements:

(1) $M$ is a $\theta$-module over $R$;

(2) $(L+N: M)=(L: M)+(N: M)$ for all saturated submodules $L$ and $N$ of $M$;

(3) $\left(L_{p}+N_{p}: M_{p}\right)=\left(L_{p}: M_{p}\right)+\left(N_{p}: M_{p}\right)$ for all submodules $L$ and $N$ of $M$ and for all prime ideals $p$ of $R$;

(4) $(L+N: M)=(L: M)+(N: M)$ for all submodules $L$ and $N$ of $M$;

(5) $M$ is a $\mu$-module over $R$.

Then $(1) \Leftrightarrow(2)$ and $(4) \Leftrightarrow(5)$.

In particular, if $M$ is a finitely generated $R$-module, then all of the above statements are equivalent. 
Proof. (1) $\Leftrightarrow(2)$ Follows from definition.

$(4) \Leftrightarrow(5)$ Follows from [22, Lemma 3.1].

(4) $\Rightarrow(2)$ Clear.

(2) $\Rightarrow(3)$ Suppose that $M$ is finitely generated. Then $M=R m_{1}+\ldots+R m_{k}$ for some $m_{i} \in M(1 \leq i \leq k)$. Let $L$ and $N$ be two submodules of $M$. First we show that $\left(S_{p}(L)+S_{p}(N): M\right)_{p}=\left((L+N)_{p}: M_{p}\right)$ for all prime ideals $p$ of $R$. Let $p$ be a prime ideal of $R$ and assume that $r / 1 \in\left(S_{p}(L)+S_{p}(N): M\right)_{p}$. It follows that $r M \subseteq S_{p}(L)+S_{p}(N)$. Thus $r m_{i}=x_{i}+y_{i}$ for some $x_{i} \in S_{p}(L), y_{i} \in S_{p}(N)(1 \leq i \leq k)$. Therefore $c_{i} x_{i} \in L$ and $d_{i} y_{i} \in N$ for some $c_{i}, d_{i} \in R \backslash p(1 \leq i \leq k)$. Now, since $c_{1} \ldots c_{k} d_{1} \ldots d_{k} r M \subseteq L+N$, we have $r / 1 \in\left((L+N)_{p}: M_{p}\right)$, as requested. Hence, by using [15, Theorem 2.1], we have

$$
\begin{aligned}
\left(L_{p}: M_{p}\right)+\left(N_{p}: M_{p}\right) & =\left(S_{p}(L): M\right)_{p}+\left(S_{p}(N): M\right)_{p} \\
& =\left(\left(S_{p}(L): M\right)+\left(S_{p}(N): M\right)\right)_{p} \\
& =\left(S_{p}(L)+S_{p}(N): M\right)_{p} \\
& =\left((L+N)_{p}: M_{p}\right) \\
& =\left(L_{p}+N_{p}: M_{p}\right) .
\end{aligned}
$$

$(3) \Rightarrow(4)$ Follows from [3, Proposition 3.8 and Corollaries 3.4 and 3.15].

(4) $\Rightarrow$ (3) Follows from [3, Corollary 3.4 and Corollary 3.15].

Corollary 2.10. For any ring $R$, every cyclic $R$-module is a $\theta$-module.

Proof. Follows from [22, Corollary 3.7] and Theorem 2.9.

Corollary 2.11. Let $M$ be a finitely generated $R$-module. Then the following statements are equivalent:

(1) $M$ is a $\theta$-module over $R$;

(2) $M_{p}$ is a $\theta$-module over $R_{p}$ for every prime ideal $p$ of $R$;

(3) $M_{m}$ is a $\theta$-module over $R_{m}$ for every maximal ideal $m$ of $R$;

(4) $M$ is a $\mu$-module over $R$;

(5) $M$ is a $\sigma$-module over $R$;

(6) $M$ is a multiplication module over $R$.

Proof. (1) $\Leftrightarrow$ (4) By Theorem 2.9.

$(4) \Leftrightarrow(5) \Leftrightarrow(6)$ By [20, Theorem 2.11 and Theorem 2.19].

$(6) \Leftrightarrow(2) \Leftrightarrow(3)$ By [4, Lemma 2 (ii)], [20, Theorem 2.11] and Theorem 2.9.

Corollary 2.12. Let $R$ be a ring. If $M$ is a finitely generated $\theta$-module over $R$ and $((0): M)=$ Re for some idempotent e of $R$, then $M$ is an $\eta$-module over $R$. In particular, every finitely generated faithful $\theta$-module is an $\eta$-module.

Proof. By Corollary 2.11 $M$ is a multiplication $R$-module, and then by [21, Theorem 11] $M$ is a projective $R$-module. Thus by Corollary 2.6, $M$ is an $\eta$-module over $R$.

Now, we investigate conditions under which $\eta$ and $\theta$ are injective or surjective.

Theorem 2.13. Let $\eta$ and $\theta$ be as before. Then

(1) $\eta \theta \eta=\eta$

(2) $\theta \eta \theta=\theta$.

Proof. (1) Let $p$ be a prime ideal of $R$ and $I \in \mathfrak{S}_{p}(R)$. Since $\eta \theta \eta(I)=S_{p}\left(\left(S_{p}(I M)\right.\right.$ : $M) M)$, we must show that $S_{p}\left(\left(S_{p}(I M): M\right) M\right)=S_{p}(I M)$. First note that, since $I \subseteq$ $\left(S_{p}(I M): M\right)$, we have $I M \subseteq\left(S_{p}(I M): M\right) M$ and thus $S_{p}(I M) \subseteq S_{p}\left(\left(S_{p}(I M): M\right) M\right)$. The reverse inclusion follows from

$$
S_{p}\left(\left(S_{p}(I M): M\right) M\right) \subseteq S_{p}\left(S_{p}(I M)\right)=S_{p}(I M) .
$$


(2) Let $p$ be a prime ideal of $R$ and $N \in \mathfrak{S}_{p}(M)$. Now, since $\theta \eta \theta(N)=\left(S_{p}((N: M) M)\right.$ : $M)$, we must show that $\left(S_{p}((N: M) M): M\right)=(N: M)$. Since $(N: M) M \subseteq S_{p}((N$ : $M) M)$, we have $(N: M) \subseteq\left(S_{p}((N: M) M): M\right)$. The reverse inclusion follows from

$$
\left(S_{p}((N: M) M): M\right) \subseteq\left(S_{p}(N): M\right)=(N: M) .
$$

Corollary 2.14. Let $\eta$ and $\theta$ be as before, and $p$ be a prime ideal of $R$. Then the following statements are equivalent:

(1) $\eta: \mathfrak{S}_{p}(R) \rightarrow \mathfrak{S}_{p}(M)$ is a surjection;

(2) $\eta \theta=1$;

(3) $S_{p}((N: M) M)=N$ for all $N \in \mathfrak{S}_{p}(M)$;

(4) $\theta: \mathfrak{S}_{p}(M) \rightarrow \mathfrak{S}_{p}(R)$ is an injection.

Proof. $(1) \Rightarrow(2)$ and $(4) \Rightarrow$ (2) follows from Theorem 2.13.

$(2) \Leftrightarrow(3),(2) \Rightarrow(1)$ and $(2) \Rightarrow(4)$ are clear.

Corollary 2.15. Let $\eta$ and $\theta$ be as before, and $p$ be a prime ideal of $R$. Then the following statements are equivalent:

(1) $\eta: \mathfrak{S}_{p}(R) \rightarrow \mathfrak{S}_{p}(M)$ is an injection;

(2) $\theta \eta=1$;

(3) $\left(S_{p}(I M): M\right)=I$ for all $I \in \mathfrak{S}_{p}(R)$;

(4) $\theta: \mathfrak{S}_{p}(M) \rightarrow \mathfrak{S}_{p}(R)$ is a surjection.

Proof. (1) $\Rightarrow(2)$ and (4) $\Rightarrow$ (2) follows from Theorem 2.13.

$(2) \Leftrightarrow(3),(2) \Rightarrow(1)$ and $(2) \Rightarrow(4)$ are clear.

Corollary 2.16. Let $\eta$ and $\theta$ be as before. Then $\eta$ is a bijection if and only if $\theta$ is a bijection. In this case $\eta$ and $\theta$ are inverse of each other.

Proof. By Corollaries 2.14 and 2.15.

Corollary 2.17. Let $R$ be a ring and $M$ be a finitely generated faithful multiplication $R$-module. Then the mappings $\eta$ and $\theta$ are lattice isomorphisms. In particular, $\eta$ and $\theta$ are inverse of each other, and therefore $\mathfrak{S}_{p}(R)$ and $\mathfrak{S}_{p}(M)$ are isomorphic lattices for all prime ideals $p$ of $R$.

Proof. Since $M$ is a faithful multiplication $R$-module, $\eta$ is an epimorphism by Theorem 2.8, and hence $\theta$ is a monomorphism by Corollary 2.14 and [22, Theorem 3.8]. On the other hand, by [15, Proposition 3.2], we have

$$
\left(S_{p}(I M): M\right)=S_{p}(I M: M)=S_{p}(I)=I,
$$

for all prime ideals $p$ of $R$ and $I \in \mathfrak{S}_{p}(R)$. Hence, by Corollary 2.15, $\eta$ is an injection and $\theta$ is a surjection. Hence $\eta$ is an isomorphism and its inverse is $\theta$.

\section{3. $\mathfrak{S}_{p}(M)$ as a Boolean algebra}

We start this section by recalling the following basic definition.

Definition 3.1. Let $R$ be a ring and $p$ be a prime ideal of $R$. An $R$-module $M$ is called a $\mathfrak{S}$-distributive module with respect to $p$, if $\mathfrak{S}_{p}(M)$ is a distributive lattice.

First note the following simple fact.

Lemma 3.2. Let $R$ be a ring, $p$ a prime ideal of $R$ and $M$ be an $R$-module. Then the following statements are equivalent:

(1) $M$ is S-distributive with respect to $p$;

(2) $K \cap S_{p}(L+N)=S_{p}((K \cap L)+(K \cap N))$ for all $K, L, N \in \mathfrak{S}_{p}(M)$; 
(3) $S_{p}(K+(L \cap N))=S_{p}(K+L) \cap S_{p}(K+N)$ for all $K, L, N \in \mathfrak{S}_{p}(M)$.

Proof. By [5, Theorem I.3.2].

The following example shows that a ring $R$ may be $\mathfrak{S}$-distributive with respect to a prime ideal and not with respect to another one.

Example 3.3. Let $R=K[X, Y]$ be the ring of polynomials with independent indeterminates $X$ and $Y$ over a field $K$. It is evident that $R$ is $\mathfrak{S}$-distributive with respect to (0), since $\mathfrak{S}_{(0)}(R)=\{(0), R\}$. However, $R$ is not $\mathfrak{S}$-distributive with respect to $m=R X+R Y$. Let $p_{1}=R X, p_{2}=R Y, p_{3}=R(X+Y)$. Since $p_{1}, p_{2}$ and $p_{3}$ are prime ideals of $R$, these ideals are saturated with respect to $m$ and hence $p_{3} \cap p_{1}$ and $p_{3} \cap p_{2}$ are saturated with respect to $m$ by Lemma 2.1 (1). Now, since $p_{3} \cap\left(p_{1}+p_{2}\right) \nsubseteq\left(p_{3} \cap p_{1}\right)+\left(p_{3} \cap p_{2}\right), R$ is not S-distributive with respect to $m$ by Lemma 3.2 .

It is remarked that some classes of $R$-modules are characterized by using the localization with respect to all prime ideal of $R$ (see for example [1]). In the next result, it is seen that the class of distributive modules has this property.

Corollary 3.4. Let $R$ be a ring and $M$ be an $R$-module. Then the following conditions are equivalent:

(1) $M$ is a distributive $R$-module;

(2) $M$ is $\mathfrak{S}$-distributive with respect to any prime ideal $p$ of $R$;

(3) $M_{p}$ is a distributive $R_{p}$-module for all prime ideals $p$ of $R$.

Proof. (1) $\Rightarrow(2)$ Let $p$ be a prime ideal of $R$ and $K, L, N \in \mathfrak{S}_{p}(M)$. By Lemma 2.1 (1) and the assumption, we have

$$
S_{p}(K+L) \cap S_{p}(K+N)=S_{p}((K+L) \cap(K+N))=S_{p}(K+(L \cap N)) .
$$

Thus, the result follows from Lemma $3.2(3)$.

(2) $\Rightarrow(3)$ Let $p$ be a prime ideal of $R$ and $K, L$ and $N$ be submodules of $M$. It suffices to show that $\left(K_{p}+L_{p}\right) \cap\left(K_{p}+N_{p}\right) \subseteq\left(K_{p}+\left(L_{p} \cap N_{p}\right)\right)$ or equivalently, by [3, Corollary 3.4], $((K+L) \cap(K+N))_{p} \subseteq(K+(L \cap N))_{p}$. For this, let $x / s \in((K+L) \cap(K+N))_{p}$. Thus there are elements $k_{1}, k_{2} \in K, l \in L, n \in N$ and $s_{1}, s_{2} \in R \backslash p$ such that $x / s=$ $\left(k_{1}+l\right) / s_{1}=\left(k_{2}+n\right) / s_{2}$. It follows that $u s s_{1} s_{2} x=\left(k_{1}+l\right)=\left(k_{2}+n\right)$ for some $u \in R \backslash p$ so that $x \in S_{p}(K+L) \cap S_{p}(K+N)$. Hence by (2), $x \in S_{p}(K+(L \cap N))$. Therefore $c x \in K+(L \cap N)$ for some $c \in R \backslash p$ which implies that $x / s=c x / c s \in(K+(L \cap N))_{p}$, as required.

$(3) \Rightarrow(1)$ Follows from [3, Corollary 3.4 and Proposition 3.8].

Corollary 3.5. Let $R$ be an arithmetical ring, and $M$ be a multiplication $R$-module. Then $M$ is a $\mathfrak{S}$-distributive $R$-module with respect to any prime ideal of $R$.

Proof. By [8, Proposition 1.2] and Corollary 3.4.

Our next example shows that $M$ being a multiplication module is needed in Corollary 3.5 .

Example 3.6. Let $K$ be a field and $V=K \oplus K$ be the usual two-dimensional vector space over $K$. It is easy to see that every subspace of $V$ is saturated with respect to (0). Now if $W_{1}=K(1,0), W_{2}=K(0,1)$ and $W_{3}=K(1,1)$. Then $W_{3} \cap\left(W_{1}+W_{2}\right)=W_{3}$ while $\left(W_{3} \cap W_{1}\right)+\left(W_{3} \cap W_{2}\right)=K(0,0)$. Thus $V$ is not $\mathfrak{S}$-distributive

We recall that a distributive lattice $(L, \vee, \wedge)$ is a Boolean algebra if there is a unary operation ' on $L$ and two constants 0 and 1 such that $x \wedge x^{\prime}=0$ and $x \vee x^{\prime}=1$.

Let $M$ be a semisimple $R$-module and $N$ a submodule of $M$. Then, by definition, there is a submodule $L$ of $M$ such that $M=N \oplus L$. We define the unary operation ' on $\mathfrak{S}_{p}(M)$ by $N^{\prime}=S_{p}(L)$. 
Theorem 3.7. Let $R$ be a semisimple ring, $p$ a prime ideal of $R$ and $M$ a distributive $R$-module. Then the lattice $\mathfrak{S}_{p}(M)$ is a Boolean algebra with the unary operation' defined above, $\mathbf{0}=S_{p}(0)$ and $\mathbf{1}=M$.

Proof. By Corollary 3.4, $M$ is a $\mathfrak{S}$-distributive $R$-module. By using Lemma 2.1 (1),

$$
N \wedge N^{\prime}=N \cap N^{\prime}=S_{p}(N) \cap S_{p}(L)=S_{p}(N \cap L)=S_{p}(0)=\mathbf{0} .
$$

Moreover, $M=N+L \subseteq S_{p}(N)+S_{p}(L) \subseteq S_{p}\left(S_{p}(N)+S_{p}(L)\right)$, which implies

$$
N \vee N^{\prime}=S_{p}\left(N+N^{\prime}\right)=S_{p}\left(S_{p}(N)+S_{p}(L)\right)=M .
$$

Hence $\mathfrak{S}_{p}(M)$ is a Boolean algebra.

From now on, $\mathfrak{S}_{p}(M)$ is assumed to be a Boolean algebra with the above assumptions.

Corollary 3.8. For any semisimple ring $R, \mathfrak{S}_{p}(R)$ is a Boolean algebra with respect to any prime ideal $p$ of $R$.

Proof. Let $R$ be a semisimple ring and $p$ a prime ideal of $R$. By [12, Exercise 1.2.5] $R$ is an arithmetical ring. Thus by Theorem $3.7, \mathfrak{S}_{p}(R)$ is a Boolean algebra.

Corollary 3.9. Let $R$ be a semisimple ring and $M$ be a distributive $R$-module. Then $\mathfrak{S}_{p}(M)$ is a Boolean ring with the following operations:

$$
L+N=S_{p}\left(L \cap S_{p}(\tilde{N})+S_{p}(\tilde{L}) \cap N\right) \text { and } L \cdot N=L \cap N,
$$

where $M=L \oplus \tilde{L}=N \oplus \tilde{N}$.

Proof. Follows from Theorem 3.7 and [5, Theorem IV.2.3].

Corollary 3.10. Let $R$ be a semisimple ring, $p$ a prime ideal of $R$ and $M$ a multiplication $R$-module. Then $M$ is cyclic and the lattice $\mathfrak{S}_{p}(M)$ is a Boolean algebra.

Proof. Since $R$ is a semisimple ring, by [12, Corollary 2.6], $R$ is an Artinian ring. Hence $M$ is cyclic by [7, Corollary 2.9]. Also, by [12, Exercise 1.2.5], $R$ is an arithmetical ring. Thus by [8, Proposition 1.2], $M$ is a distributive $R$-module. Hence by Theorem $3.7, \mathfrak{S}_{p}(M)$ is a Boolean algebra with respect to any prime ideal $p$ of $R$.

Theorem 3.11. Let $R$ be a ring, $p$ a prime ideal of $R, M$ an $R$-module and $N$ a submodule of $M$. Then the followings hold:

(1) For any submodule $L$ containing $N, S_{p}(L / N)=S_{p}(L) / N$. In particular, the assignment $L \mapsto L / N$ is a one to one corresponding between the set $\{L \mid L \in$ $\left.\mathfrak{S}_{p}(M), L \supseteq N\right\}$ and $\mathfrak{S}_{p}(M / N)$;

(2) If $M$ is a $\mathfrak{S}$-distributive lattice over $R$ with respect to $p$, then $M / N$ is $\mathfrak{S}$-distributive over $R$ with respect to $p$;

(3) If $R$ is a semisimple ring and $M$ a distributive $R$-module, then $\mathfrak{S}_{p}(M / N)$ is a Boolean algebra.

Proof. (1) Clear.

(2) Let $\mathfrak{S}_{p}(M)$ be a distributive lattice with the operations $\vee$ and $\wedge$ and $\mathfrak{S}_{p}(M / N)$ be a lattice with the operations $\tilde{V}$ and $\tilde{\Lambda}$. It is seen that $\tilde{V}$ and $\tilde{\Lambda}$ are expressed by $\vee$ and $\wedge$ respectively as follows:

$$
\begin{aligned}
L / N \tilde{\nabla} K / N & =S_{p}(L / N+K / N) \\
& =S_{p}((L+K) / N) \\
& =S_{p}(L+K) / N \\
& =(L \vee K) / N,
\end{aligned}
$$

and

$$
L / N \tilde{\wedge} K / N=L / N \cap K / N=(L \cap K) / N=(L \wedge K) / N .
$$


By these statements, the distributivity of $\mathfrak{S}_{p}(M / N)$ follows immediately from the distributivity of $\mathfrak{S}_{p}(M)$.

(3) Follows from Theorem 3.7 and (2).

Theorem 3.12. Let $R$ be a ring, $T$ a multiplicatively closed subset of $R, M$ an $R$-module and $N$ a submodule of $M$. Then the followings hold:

(1) $S_{T^{-1} p}\left(T^{-1} N\right)=T^{-1}\left(S_{p}(N)\right)$ for all prime ideals $p$ disjoint from $T$. In particular, $N \in \mathfrak{S}_{p}(M)$ if and only if $T^{-1} N \in \mathfrak{S}_{T^{-1} p}\left(T^{-1} M\right)$ for all prime ideals $p$ disjoint from $T$;

(2) If $M$ is a $\mathfrak{S}$-distributive lattice over $R$ with respect to a prime ideal $p$ of $R$ such that $p \cap T=\emptyset$, then $T^{-1} M$ is $\mathfrak{S}$-distributive over $T^{-1} R$ with respect to $T^{-1} p$;

(3) If $R$ is a semisimple ring, $p$ a prime ideal of $R$ with $p \cap T=\emptyset$ and $M$ a distributive $R$-module, then $\mathfrak{S}_{T^{-1} p}\left(T^{-1} M\right)$ is a Boolean algebra.

Proof. (1) Clear.

(2) Let $p$ be a prime ideal of $R$ such that $p \cap T=\emptyset$. Let $\mathfrak{S}_{p}(M)$ be a distributive lattice with the operations $\vee$ and $\wedge$ and $\mathfrak{S}_{T^{-1} p}\left(T^{-1} M\right)$ be a lattice with the operations $\tilde{V}$ and $\tilde{\wedge}$. It is seen that $\tilde{V}$ and $\tilde{\wedge}$ are expressed by $\vee$ and $\wedge$ respectively as follows:

$$
\begin{aligned}
T^{-1} L \tilde{\vee} T^{-1} N & =S_{T^{-1} p}\left(T^{-1} L+T^{-1} N\right) \\
& =S_{T^{-1} p}\left(T^{-1}(L+N)\right) \\
& =T^{-1}\left(S_{p}(L+N)\right) \\
& =T^{-1}(L \vee N),
\end{aligned}
$$

and

$$
\begin{aligned}
T^{-1} L \tilde{\wedge} T^{-1} N & =T^{-1} L \cap T^{-1} N \\
& =T^{-1}(L \cap N) \\
& =T^{-1}(L \wedge N) .
\end{aligned}
$$

By these statements, the distributivity of $\mathfrak{S}_{T^{-1}} p\left(T^{-1} M\right)$ follows immediately from the distributivity of $\mathfrak{S}_{p}(M)$.

(3) Since $R$ is a semisimple ring, then so is $T^{-1} R$. Thus the result follows from Theorem 3.7 and (2).

Let $A$ and $B$ be Boolean algebras. A function $f: A \rightarrow B$ is called a Boolean algebra homomorphism, if $f$ is a lattice homomorphism, $f(\mathbf{0})=\mathbf{0}, f(\mathbf{1})=\mathbf{1}$ and $f\left(a^{\prime}\right)=f(a)^{\prime}$ for all $a \in A$. It is easily proved that a lattice homomorphism $f$ preserves $\mathbf{0}$ and $\mathbf{1}$ if and only if it preserves '. Thus, in order to show that a function $f$ between two Boolean algebras is a Boolean algebra homomorphism, it suffices to check that $f$ preserves lattice operations $\vee$ and $\wedge$ and constants $\mathbf{0 , 1}$.

Theorem 3.13. Let $R$ be a semisimple ring, $p$ a prime ideal of $R$ and $M$ a distributive $R$-module. Then $\eta: \mathfrak{S}_{p}(R) \rightarrow \mathfrak{S}_{p}(M)$ is a Boolean algebra homomorphism.

Proof. First note that $\mathfrak{S}_{p}(M)$ and $\mathfrak{S}_{p}(R)$ are Boolean algebras, by Theorem 3.7 and Corollary 3.8 respectively. By Corollary 2.7, $\eta$ is a lattice homomorphism. Also,

$$
\eta(\mathbf{0})=\eta\left(S_{p}(0)\right)=S_{p}\left(S_{p}(0) M\right)=S_{p}(0)=\mathbf{0},
$$

and

$$
\eta(\mathbf{1})=\eta(R)=S_{p}(R M)=S_{p}(M)=M=\mathbf{1} .
$$

Hence, as noted above, $\eta$ is a Boolean algebra homomorphism.

Corollary 3.14. Let $R$ be a semisimple ring, $p$ a prime ideal of $R$ and $M$ a multiplication $R$-module. Then $\eta: \mathfrak{S}_{p}(R) \rightarrow \mathfrak{S}_{p}(M)$ is a Boolean algebra epimorphism. 
Proof. By Corollaries 3.8 and $3.10, \mathfrak{S}_{p}(R)$ and $\mathfrak{S}_{p}(M)$ are Boolean algebras respectively. Also, by the proof of Corollary 3.10, $M$ is distributive. Thus by Theorem 3.13, $\eta$ is a Boolean algebra homomorphism. Moreover, if $N \in \mathfrak{S}_{p}(M)$, then $(N: M) \in \mathfrak{S}_{p}(R)$ and

$$
\eta(N: M)=S_{p}((N: M) M)=S_{p}(N)=N .
$$

Thus, $\eta$ is an epimorphism.

Finally, we remark that if $M$ is a faithful multiplication module over a semisimple ring $R$, then since $M$ is cyclic by Corollary 3.10, we conclude that $M$ is isomorphic to $R$. So it clearly follows that $\eta$ and $\theta$ are Boolean algebra isomorphisms.

Acknowledgment. The authors would like to thank the referee for his/her helpful comments.

\section{References}

[1] M. Alkan and Y. Tiras, On invertible and dense submodules, Comm. Algebra, 32 (10), 3911-3919, 2004.

[2] M. Alkan and Y. Tiras, On prime submodules, Rocky Mountain J. Math. 37 (3), 709-722, 2007.

[3] M.F. Atiyah and I.G. Macdonald, Introduction to Commutative Algebra, AddisonWesley, London, 1969.

[4] A. Barnard, Multiplication modules, J. Algebra, 71 (1), 174-178, 1981.

[5] S. Burris and H.P. Sankappanavar, A Course in Universal Algebra, Springer-Verlag, New York, 1981.

[6] J. Dauns, Prime submodules, J. Reine Angew. Math. 298, 156-181, 1978.

[7] Z.A. El-Bast and P.F. Smith, Multiplication modules, Comm. Algebra, 16 (4), 755799, 1988.

[8] V. Erdogdu, Multiplication modules which are distributive, J. Pure Appl. Algebra, 54, 209-213, 1988.

[9] J.B. Harehdashti and H.F. Moghimi, Complete homomorphisms between the lattices of radical submodules, Math. Rep. 20(70) (2), 187-200, 2018.

[10] T.W. Hungerford, Algebra, Springer-Verlag, New York, 1974.

[11] J. Jenkins and P.F. Smith, On the prime radical of a module over a commutative ring, Comm. Algebra, 20 (12), 3593-3602, 1992.

[12] T.Y. Lam, A First Course in Noncommutative Rings, Springer-Verlag, New York, 1991.

[13] M.D. Larsen and P.J. McCarthy, Multiplicative Theory of Ideals, Academic Press, New York, 1971.

[14] C.P. Lu, M-radical of submodules in modules. Math. Japonica, 34 (2), 211-219, 1989.

[15] C.P. Lu, Saturations of submodules, Comm. Algebra, 31 (6), 2655-2673, 2003.

[16] C.P. Lu, A module whose prime spectrum has the surjective natural map, Houston J. Math. 33 (1), 125-143, 2007.

[17] R.L. McCasland and M.E. Moore, On radicals of submodules, Comm. Algebra, 19 (5), 1327-1341, 1991.

[18] R.L. McCasland and M.E. Moore, Prime submodules, Comm. Algebra, 20 (6), 18031817, 1992.

[19] R.L. McCasland, M.E. Moore and P.F. Smith, On the spectrum of a Module over a commutative ring, Comm. Algebra, 25 (1), 79-103, 1997.

[20] H.F. Moghimi and J.B. Harehdashti, Mappings between lattices of radical submodules, Int. Electron. J. Algebra, 19, 35-48, 2016.

[21] P.F. Smith, Some remarks on multiplication modules, Arch. Math. 50, 223-235, 1988. 
[22] P.F. Smith, Mappings between module lattices, Int. Electron. J. Algebra, 15, 173-195, 2014.

[23] P.F. Smith, Complete homomorphisms between module lattices, Int. Electron. J. Algebra, 16, 16-31, 2014.

[24] P.F. Smith, Anti-homomorphisms between module lattices, J. Commut. Algebra, 7, 567-591, 2015. 